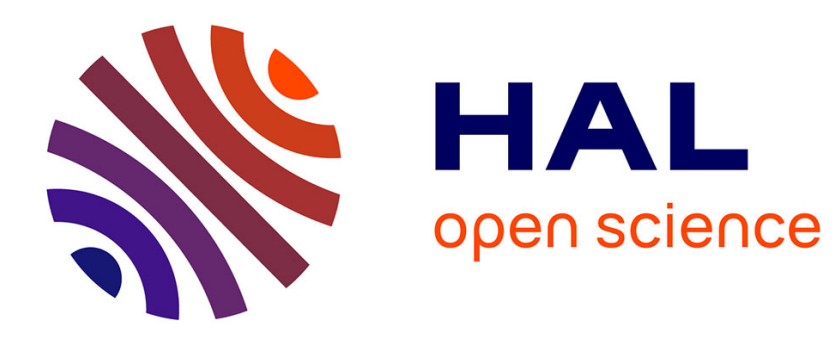

\title{
Functions, organization and etiology. A reply to Artiga and Martinez
}

\author{
Matteo Mossio, Cristian Saborido
}

\section{To cite this version:}

Matteo Mossio, Cristian Saborido. Functions, organization and etiology. A reply to Artiga and Martinez. Acta Biotheoretica, 2016, 64 (3), pp.263-275. 10.1007/s10441-016-9283-2 . hal-01354349

\author{
HAL Id: hal-01354349 \\ https://hal.science/hal-01354349
}

Submitted on 18 Aug 2016

HAL is a multi-disciplinary open access archive for the deposit and dissemination of scientific research documents, whether they are published or not. The documents may come from teaching and research institutions in France or abroad, or from public or private research centers.
L'archive ouverte pluridisciplinaire HAL, est destinée au dépôt et à la diffusion de documents scientifiques de niveau recherche, publiés ou non, émanant des établissements d'enseignement et de recherche français ou étrangers, des laboratoires publics ou privés.

\section{다)(1) $(5$}

Distributed under a Creative Commons Attribution - NonCommerciall 4.0 International 


\title{
Functions, organization and etiology \\ A reply to Artiga and Martinez
}

\author{
Published in Acta Biotheoretica \\ The final publication is available at Springer via: \\ http://link.springer.com/article/10.1007/s10441-016-9283-2
}

\begin{abstract}
Matteo Mossio
Institut d'Histoire et de Philosophie des Sciences et des Techniques (IHPST)

CNRS/Paris 1/ENS, Paris, France

matteo.mossio@univ-paris1.fr

$+33(0) 143546036$
\end{abstract}

Cristian Saborido

Department of Logic, History and Philosophy of Science, UNED, Madrid, Spain

\begin{abstract}
We reply to Artiga and Martinez's claim according to which the organizational account of cross-generation functions implies a backward looking interpretation of etiology, just as standard etiological theories of function do. We argue that Artiga and Martinez's claim stems from a fundamental misunderstanding about the notion of "closure", on which the organizational account relies. In particular, they incorrectly assume that the system, which is relevant for ascribing cross-generation organizational function, is the lineage. In contrast, we recall that organizational closure refers to a relational description of a network of mutual dependencies, abstracted from time, in which production relations are irrelevant. From an organizational perspective, ascribing a function to an entity means locating it in the abstract system that realizes closure. In particular, the position of each entity within the relational system conveys an etiological explanation of its existence, because of its dependence on the effects exerted by other entities subject to closure. Because of the abstract relational nature of closure, we maintain that the organizational account of functions does not endorse a backward looking interpretation of etiology. As a consequence, it does not fall prey of epiphenomenalism.
\end{abstract}

\section{Keywords}

Biological functions, Organization, Closure, Etiology, Epiphenomenalism 
It is always an honor when some colleagues show interest in your work and make the effort to critically appraise and improve your ideas. In "The Organizational Account of Functions is an Etiological Account of Functions" Artiga and Martinez analyze the organizational account of the notion of biological function (introduced mainly in Mossio et al. 2009 and Saborido et al. 2011, and partly enriched in Moreno \& Mossio 2015), according to which a biological trait is functional if and only if it is subject to organizational closure in the system to which it belongs.

Artiga and Martinez' analysis primarily focuses on cross-generation functions, and leads to the conclusion that the organizational account (OA) does not convey any clear substantive advantage over the classical etiological approaches, such as Millikan's (1989), Neander's (1991) or Godfrey-Smith's (1987). In their opinion, the OA simply consists in another etiological definition of function which is even "less appealing than other etiological alternatives" (Artiga \& Martinez 2016, p. 116). Since the OA aims at overcoming some of the central weaknesses of classical etiological accounts, this is indeed a strong criticism, which calls for a reply.

\section{What is the criticism about?}

Let us begin by pointing out that there is no debate or disagreement on whether the OA possesses an etiological dimension. Indeed, we have explicitly claimed in previous writings that the OA is an etiological account, insofar as it constitutes an interpretation of Wright's original definition, according to which:

"The function of $\mathrm{X}$ is $\mathrm{Z}$ means:

(1) $\mathrm{X}$ is there because it does $\mathrm{Z}$;

(2) $\mathrm{Z}$ is a consequence (or result) of X's being there" (Wright 1973 p. 48).

In contrast, we have argued that the OA presents some notable advantages over classical etiological accounts, because it integrates central insights of the systemic-dispositional theories of functions (Saborido 2014).

According to the OA, a function is a contribution of a biological trait to the maintenance of the whole organization that, in turn, contributes to maintain the trait itself (see below for more details). Accordingly, the OA puts emphasis on two features of biological functions. On the one hand, functions are dispositions of parts that contribute to a systemic capacity. In this respect, the OA involves a "systemic stance" on functional ascriptions, in line with the ideas put forward by dispositional theorists such as Cummins (1975) and Davies (2001). On the other hand, however, not any disposition is functional: functions can be ascribed only to those effects that, by contributing to the maintenance of the overall organization, etiologically determine the conditions of existence of function bearers. Organizational functions are therefore both dispositional and etiological.

In order to keep together (current) dispositions and etiology, the OA relies on the characterization of a regime of mutual dependence (that we label "closure") between the constitutive parts of biological systems. Accordingly, we have argued that the OA provides a definition of function that, while being etiological, does not fall into one of the most problematic aspects of many etiological accounts, i.e. its epiphenomenal character. In particular, we have argued that:

The crucial drawback of the [classical etiological] theories' explanatory line is the implication that functional attributions have no relation to the current contribution of the 
trait to the system, since they point solely to the selective history of the trait. This implication is problematic because it is at odds with the fact that functional attributions to biological structures do seem to have some relation-captured by the dispositional approaches - to what they currently do, and not only to what explains their current existence. Accordingly, [these] theories provide an account that is problematically epiphenomenal, in the sense that it maintains that the attribution of a function does not provide information about the 'phenomenon' (the current system) being observed. From this perspective, a function does not describe anything about the current organization of the system being analyzed. (Mossio et al. 2009, p. 821)

In contrast with other etiological accounts, which put forward an understanding of etiology by appealing to natural selection, the OA grounds etiology within the organization of each individual organism. Therefore, we have argued, the OA avoids epiphenomenalism.

In their paper, however, Artiga and Martinez contend that the OA does carry on an epiphenomenal way of thinking functions. In spite of our intentions, they argue, the OA involves a backward looking interpretation of etiology, which makes the OA a version of the Selected Effects accounts of function, such as those proposed by Millikan and Neander:

"the organizational account defended by Mossio and colleagues is, at bottom, a deployment of the distinctly historical kind of etiological insight that informs many of the best known extant accounts of function" (Artiga \& Martinez, 2016, p. 116).

Artiga and Martinez argue that the epiphenomenal character of OA is especially clear in the way it deals with cross-generation functions.

In Saborido et al. (2011), indeed, we have addressed the seemingly problematic case of those functional traits that overcome the limits of individual organisms. In particular, we have proposed an organizational interpretation of functions ascribed to cross-generation traits, i.e. traits (such as, for instance, the semen and the ovum) that have functional effects in systems different from those that produce them. As a result, there seems to be no single organization in which the trait is subject to closure. For instance, the most relevant effect of semen is not a contribution to the maintenance of the organism that produces it, but to the production of other organisms with a distinct organization (the offspring). That seems to force the advocates of the OA to choose between two quite unsatisfactory options: either they concede that cross-generation traits are not functional from an organizational perspective, or they embrace a "splitting account" - in the sense of Delancey (2006) - according to which there would be two different definitions of function, one for intra-organismic traits and other one for cross-generational ones.

In Saborido et al. (2011) we have argued that it is possible to maintain a unitary organizational account, which applies to both intra- and cross-generation functions. Our proposal relies on the idea that cross-generation functions are subject to organizational closure just as intra-organismal ones; yet, the system that realizes the relevant closure goes beyond the boundaries of individual organisms. More precisely, we have proposed to focus on what we label an organizationally closed "encompassing system" that would notably include the reproducer and the reproduced organism:

"If, in a relevant sense, a given system or a given temporal instance of a system possesses a constitutive organization because of its causal and material connection with a previous instance possessing the same organization, then it can be claimed that the two systems or the two instances are in fact instances of the same encompassing organization. In particular, the same is also true if the transmitted organization is a self-maintaining organization. [...] Since the encompassing system composed by the producer and 
reproduced organism possesses a (temporally wider) self-maintaining organization, reproductive traits are subject to organizational closure, and their functions are correctly grounded in the organizational account”. (Saborido et al. 2011, p. 599-600)

Artiga and Martinez' main criticism is that the organizational account of cross-generation functions is problematic because it implies a backward looking interpretation of etiology. According to them, the "encompassing system" invoked by the OA to ground crossgeneration functions corresponds to the notion of lineage, which is nothing but a temporal succession of organisms, and not an actual (organized) system itself. Because of this, the charge of epiphenomenalism that we address to other etiological accounts equally applies to ours. They unfold their argument by discussing how the OA would ground (in their view) the cross-generation function of donkey semen:

"Function Grounding OA:

(C1) Donkey semen has contributed to the maintenance of the organization of a donkey lineage (by aiding donkey reproduction across generations);

(C2) Semen is maintained and produced by this organization (say, its way of being a lineage, in which some individuals are parents or offspring of others);

That is, the fact that warrants our attribution of function to a cross-generational trait such as semen is that semen has contributed to donkey reproduction in the past $(\mathrm{C} 1)$ and this, in turn, explains that semen exists (C2). This is exactly the kind of function-endowing fact that etiological theories of biological function postulate" (Artiga \& Martinez, 2016, p. 112)

If their argument were correct, they would be right in claiming the OA is not clearly innovative with respect to other etiological accounts, at least with regards to cross-generation functions. In particular, it would suffer from the same problem of epiphenomenalism.

Yet, we think that their entire argument is undermined by an incorrect interpretation of some of the central tenets of the organizational account. In particular, they do not correctly understand what the pivotal notion of organizational closure is about. In the following section, we explain in some details what the misinterpretation consists in. It is worth noting that we do not introduce new ideas in this paper, but we rather elaborate on those already exposed in the papers examined by Artiga and Martinez. Yet, the very fact that Artiga and Martinez formulated their criticism suggests that some aspects of the OA may require a clearer formulation. This is what we aim at in the following sections.

\section{What is organizational closure?}

Let us recall the organizational definition of biological functions, which implies the fulfillment of three different conditions. According to the OA, a trait $\mathrm{T}$ has (or serves) a function $\mathrm{F}$ within the organization $\mathrm{O}$ of a system $\mathrm{S}$ if and only if ${ }^{1}$ :

$\mathrm{C} 1$. T exerts a constraint that contributes to the maintenance of $\mathrm{O}$ of $\mathrm{S}$;

$\mathrm{C} 2 . \mathrm{T}$ is maintained under some constraints exerted by $\mathrm{O}$;

C3. S realizes organizational closure.

1 This definition comes from Moreno \& Mossio (2015) and slightly amends that provided in Saborido et al., (2011). In particular, it makes explicit that functions are to be conceived of as constraints exerted on processes and reactions. See below for more. 
As C3 illustrates, the idea of organizational closure plays a crucial role. In a nutshell, closure

"designates a feature of biological systems by virtue of which their constitutive components and operations depend on each other for their production and maintenance and, moreover, collectively contribute to determining the conditions under which the system itself can exist" (Moreno \& Mossio 2015: p. 36, see also Mossio 2013).

In particular, we have recently put forward a more formal characterization (Montévil \& Mossio, 2015), which describes closure as the mutual dependence among a set of entities acting as constraints on the thermodynamic flow of energy and matter (processes and reactions) traversing the system. Organizational closure should therefore be understood as a "closure of constraints": what is "organized" in biology, we hold, are the constraints that constitute the system and control its dynamics.

Leaving aside technical details, which are not relevant here, it seems that Artiga and Martinez have difficulties in getting a clear understanding of the very notion of closure and, more precisely, of its theoretical nature. When discussing how the OA ascribes cross-generation functions, they note:

"the idea [of OA] is that there is, after all, an organization that underpins the attribution of function to cross-generational traits: the "encompassing system" which includes (at least) the system that carries the trait and its offspring in the following generation. To a first approximation, then, an encompassing system includes a set of organisms of different generations of a single lineage". (Artiga and Martinez, 2016, p.110)

So far so good. Troubles begin when Artiga and Martinez interpret the "includes" above as the idea that the encompassing system is the (relevant fraction of the) lineage. It is true, as Artiga and Martinez remind, that we pointed out the following in our 2011 paper:

"The crucial point is that the organization of the system constituted by the conjunction of the reproducing and reproduced organisms (in this specific case, a minimal lineage with two elements) has exactly the same status, in terms of self-maintenance, as that of the individual organisms" (Saborido et al. 2011 p. 600).

This sentence might appear ambiguous at first sight. However, it by no means implies the idea that the encompassing organized system is equivalent to the lineage. It rather means that the encompassing organized system can be described by considering jointly a set of organisms, which in other respects constitute a lineage. Yet, the kind of relations among these organisms that matters for describing the two systems (the organized system vs. the lineage) is distinct.

To better understand this fundamental point, let us focus on closure as a general notion. Organizational closure consists in a regime in which a set of entities (acting as constraints) realizes a mutual dependence, such that the conditions of existence of each of them depend (at least in part) on the effects produced by (at least) one other constraint included in the set. Therefore, closure implies a circular relation between the mutually dependent constraints; as such, we submit, it can only be described by abstracting from the physical time. As a circular regime, indeed, closure cannot unfold in time and requires an abstract relational description.

To see why, consider the mutual dependence between the heart and the lungs in a mammal (both the heart and the lungs complying, by hypothesis, with our characterization of a constraint): the heart depends on the lungs and, reciprocally, the lungs depend on the heart. How is this mutual dependence realized in the physical world? In a real organism, we would observe a chain of dependences unfolding in time: for instance, the heart would contribute at 
time $t_{1}$ to maintaining the lungs, which would contribute at $t_{2}$ to maintaining the heart, which would contribute to maintaining the lungs at time $t_{3}$ and so on $^{2}$. To interpret such a linear succession of events as a closed regime, an additional theoretical move is required. In particular, we must make the hypothesis that, for instance, the heart at $t_{1}$ and $t_{3}$ is, in some relevant sense, the same heart. If so, we could conclude that the heart maintains the lungs and, reciprocally, that the lungs maintain the (same) heart: as a result we obtain mutual dependence between them, and both the heart and the lungs become functional, according to the organizational account. Now, on what ground can we posit that two temporal instances of the heart are actually the same heart? One good reason could the fact that many similarities exist between the description of the heart as a constraint at $t_{1}$ and $t_{3}$, as well as the kind of dependence that it has with the lungs at $t_{1}$ and $t_{3}$. In any case, we submit that the identification cannot mean that the heart at $t_{3}$ is the same temporal instance than the heart at $t_{1}$ because this would imply that the chain of dependences has gone back in time. In this respect, the background assumption is that a scientifically acceptable characterisation of a physical circular regime should not reverse the "arrow of time".

As a consequence, the very idea of closure makes sense only by identifying similar patterns in the chain of constraint dependences through time, and by identifying these patterns as the same by neglecting the fact that they occurred in different moments. For this reason, we claim that the description of closure requires abstracting from the flow of time. In the simplified example above, for instance, the mutual dependence between the lungs and the heart (the heart depends on the lungs and vice-versa) does not make any reference to the temporal unfolding of the constraints exerted by each entity. What matters is only their abstract relational dependence, while time is neglected for theoretical reasons that pertain to the scientific understanding of circularities: there cannot be circular regimes in the physical time.

The very same theoretical approach applies to cross- generation organizations. To describe them, the OA takes into account a chain of constraint dependences that unfolds in time beyond the boundaries of a single generation. In the chain, the iteration of similar patterns can be identified across generation, as for instance the fact that the constraint exerted by the heart in a mammal, as well as the way in which it maintains the lungs, is very similar in the offspring and parents. The same reasoning can of course be applied to many other constraints at work in the organisms. Consequently, one can conclude that a set of constraints realizes a closure, an "encompassing" self-maintaining organization.

Crucially, the fact that from the very same chain of dependences one could also describe a succession of distinct organisms linked by a production relation is totally irrelevant here: their being a sequence of reproducers and reproduced systems, which eventually generates a lineage, plays no role in ascribing cross-generation functions from an organizational perspective. In the encompassing organization, there are neither reproducers nor reproduced systems: there is only one self-maintaining organization that maintains (what could in other respects be called cross-generation) functions (for example, the functions of the sperm and the ovum) and is maintained by them - just as any individual organism maintains its functional constraints and is maintained by them. What matters for describing the encompassing self-maintaining organization is the continuity of constraints dependences and the iteration of similar patters which allow folding up the chain, while the discontinuity which is relevant for describing the production relation among them (the reproduction) does not. As a consequence, we maintain that the encompassing self-maintaining system invoked

\footnotetext{
${ }^{2}$ This is of course a simplification of the real situation. Among other things, there may be a partial overlap between the time scales $t_{1} t_{2}$ and $t_{3}$.
} 
by the OA is not an abstraction of biological kinships such as species or lineages. On the contrary, it is characterized by the fact of instantiating organizational closure just as individual biological organisms do (although this does not imply that these encompassing systems are organisms themselves) ${ }^{3}$.

The difficulty to adequately understanding what organizational closure is about has induced Artiga and Martinez' incorrect interpretation of the encompassing organization as a lineage; in turn, this misinterpretation has paved the way to their main criticism, the idea that the OA endorses a backward looking interpretation of etiology.

Before answering to their criticism, let us first deal with a specific point that Artiga and Martinez raise in their paper. The point refers to the "period of time" which is necessary for describing closure. According to Artiga and Martinez, "[ascribing an organizational function to the donkey semen] can only mean that the semen in question must have contributed to the maintenance of the donkey lineage also in earlier generations" (Artiga and Martinez 2016, p. 112): even in the OA - they submit - traits must produce their effects for a certain period of time to acquire a functions.

In the OA, however, this does mean something else. To ascribe functions, the OA must first describe the organizational closure of the organism under scrutiny, which in turn requires picking up the relevant constraints. Now, as discussed above, biological constraints and their dependences unfold in time. Accordingly, the period of time evoked by Artiga and Martinez is the time duration that must be considered for observing the relevant portion of the chain of constraint dependences, and the iteration of similarities. In our toy example above, the chain of dependences must unfold at least until $t_{3}$, when an iteration of the dependence between the heart and the lungs, similar to that observed at $t_{1}$, occurs. Once that that duration has elapsed, and the constraints have been exerted, their mutual dependencies and overall closure can be described by abstracting them from physical time, as recalled above. Therefore, and in sharp contrast to Artiga and Martinez' claim, some time is required for describing closure, but not for ascribing functions. Once closure is described, functions are ipso facto ascribed to all organized constraints, with no consideration for the fact that functions might or not have been performed for a short or long time, or in earlier generations.

Let us also emphasize that when we claim that functional traits must be produced and maintained by the system we simply mean that they must exist and last long enough to exert the constraint: an enzyme which would deteriorate too fast would be unable to catalyze chemical reactions. But this condition, against what Artiga and Martinez suggest (Artiga and Martinez, 2016, p. 111), holds even for possible one-shot functions, i.e. functions performed only once in the history of life, and possibly for a very short time. A constraint must exist long enough to perform its function, and therefore to be able to produce an effect which contributes to the maintenance of the organization and, thereby, of the function bearer. Yet, the frequency or duration of functional effects is per se irrelevant.

In our view, in sum, Artiga and Martinez' misinterpretation of the very concept of organizational closure has generated a systematic bias in their reading and understanding of the organizational account of functions. Let us now come back to their main point: the claim that the organizational account of cross-generation functions implies a backward looking interpretation of etiology.

\footnotetext{
${ }^{3}$ As a matter of fact, the organizational account could possibly be applied to describe other kinds of closed organizations that include individual organisms (such as social or ecological ones, see for instance Nunes Neto et al. 2014), and ascribe functions to their parts.
} 


\section{Is the organizational account a backward looking one?}

By relying on the clarifications provided in the previous section, we reply to Artiga and Martinez' main criticism by firmly maintaining that the OA does not imply per se a temporal interpretation of the etiological dimension, and in particular not a backward looking one.

The OA ascribe functions to constraints subject to organizational closure that, as discussed, is described by abstracting from time the relevant set of mutual dependencies. Because of the very nature of organizational closure as a theoretical construct, hence, functional ascriptions have no temporal orientation. From an organizational perspective, ascribing a function to a constraint means locating it in an abstract relational system that realizes closure. In particular, the position of each constraint within the organization conveys a relational explanation of its existence (its maintenance), because of its dependence on the effects exerted by other constraints. Accordingly, the etiological dimension of functions consists in the fact that organized constraints are not only the origin, but also the target of abstract dependence relations in a closed system. As a consequence, for each constraint subject to closure, what explain etiologically its maintenance $(\mathrm{C} 2)$ is not supposed to temporally precede its contribution to the maintenance of the system $(\mathrm{C} 1)$. There is no temporal ordering between $\mathrm{C} 1$ and $\mathrm{C} 2$; they differ uniquely with respect to the orientation of the dependence relation (the arrow) in the relational graph. To refer again to our example above, the mutual dependence between the heart and the lungs (and more generally between the whole set of constraints subject to closure in a mammal) has no temporal orientation as such. In particular, what contributes to explain etiologically the maintenance of the heart (here, the constraint exerted by the lungs) does not precede or follow its contribution as a constraint.

Of course, the abstract relational character of organizational functions does not mean that one cannot provide a temporal interpretation for each specific situation. Indeed, it might be the case that, for a specific constraint, $\mathrm{C} 1$ follows temporally $\mathrm{C} 2$. Yet, this is just one possibility, the other being that $\mathrm{C} 1$ temporally precedes $\mathrm{C} 2$, in such a way that the maintenance of the trait is a potential course of actions to be realized in the future. Just as for Wright's definition, hence, the organizational account supports both backward looking and forward looking interpretations of etiology (the latter having been championed, in particular, by Bigelow \& Pargetter 1987, and Walsh 1996). In fact, the expression "is there" in Wright's formulation can be read in a wider sense and it can refer to future ("X will be there") and even counterfactual states ("X would be there"). All these interpretations are compatible with the OA. In all cases, again, the temporal ordering is as such irrelevant for functional ascriptions from the organizational perspective.

As a consequence, we do maintain that the OA, insofar as it does not endorse a backward looking interpretation of etiology, does not fall prey of epiphenomenalism. Indeed, functions are epiphenomenal when they convey an etiological dimension, which refers to some state of affairs having occurred in the past. Accordingly, an effect produced by a trait (type) is functional if it has contributed to set the conditions of existence of the trait in the past: what the trait (token) does here and now is not relevant for function ascriptions.

In turn, the OA interprets the etiological dimension of functions as the fact of being the target of dependence relations in an abstract graph of organizational closure. What explains the existence of the function bearer is the relational structure of the organization (and the related network of constraints dependencies), which does not involve any temporality. To build such a relational structure, the OA does not need to restrict the relevant chains of dependences to those having occurred in the past: in particular, there is no reason to exclude the current contribution of a trait, insofar as such current contribution can be subject to closure with other contributions occurring simultaneously, in the past, or in the future. For instance, one 
may say that the lungs of a mammal have contributed a second ago to maintaining the heart, which contributes now to maintaining the lungs. As above, if we have good reasons to identifying the lungs that we observed a second ago with the lungs that we observe now, then we can conclude that the current contribution of the heart is subject to organizational closure. Since there is no theoretical reason for excluding current effects of traits from the description of abstract closure, we hold that, by construction organizational functions are not epiphenomenal.

Let us insist on this: one thing are the conditions in which functions are ascribed, and another thing are the conditions in which functions are performed. In an organism, functions are performed in time, and their dependencies unfold in time; yet, one of the original insights of the $\mathrm{OA}$ is that such temporal ordering does not play a role in ascribing biological functions. As a consequence, the contribution of a trait to its own existence does not need to precede its own existence in time: in particular, one can appeal to the current contribution of a trait to account for its own existence. Unfortunately, such a distinction gets lost in Artiga and Martinez's interpretation.

Lastly, Artiga and Martinez hold that, if the OA were not epiphenomenal it would unable to give an account of malfunctions:

"[OA] be unable to account for malfunction since it would be impossible for a trait to have a function and fail to contribute to a system" (Artiga and Martinez, 2016, p. 113).

Although we cannot discuss this issue in details here, we would mention that the OA has recently started to address the notion of malfunction (Saborido \& Moreno 2015). The underlying idea is that, in spite to what most advocates of selected effects theories claim, there are ways of accounting how a trait can "fail" to perform a function without adopting an epiphenomenal stance.

\section{How are organizational functions individuated?}

In the final part of their paper, Artiga and Martinez critically examine how the OA provides criteria to individuate functional traits. According to them, the fact that the OA aims at ascribing functions to particular traits because of their role in the organized system means that the OA is committed to the idea that functions are ascribed to traits tokens and not to trait types. Artiga and Martinez argue that this position implies that:

"the different instances of semen of many individual donkeys constitute the same token. That is, the organizational theorist must hold that the semen of a significant set of donkeys that belong to different generations are numerically the same semen" (Artiga and Martinez 2016, p. 114).

Indeed, the OA claims that the instances of cross-generation traits are not subject to closure within individual organisms at each generation, but only within the encompassing closed organization. Therefore, we do submit that these instances are functionally individuated as the same trait. Artiga and Martinez conclude:

"this way of individuating traits is extremely counterintuitive - outlandish, rather" (Artiga and Martinez 2016, p. 115).

Our reply is twofold. On the one hand, we think that the question whether the OA ascribes functions to tokens or types is a general one, which applies to both intra- and crossgeneration traits. Indeed, one might wonder whether any intra-generation functional trait is a 
token or a type (instantiated by several temporal instances), just as Artiga and Martinez question whether the semen of the reproducer and the semen of the reproduced donkey are a single token or two tokens belonging to the same type. Yet, the answer to that question depends on background metaphysical assumptions that seem to us quite irrelevant for the purpose of ascribing functions. Whether or not a man's heart or semen is a token or a type might possibly play a role for dealing with biological malfunctions, but not for individuating functional traits. The two issues are related but logically distinct: accordingly, we hold that the criteria of individuation provided by the OA can be adopted even though the token/type issue is not settled and left open.

On the other hand, we maintain that the OA applies the very same criterion of individuation of functional traits both for intra-generation and cross-generation ones. In both cases, temporal instances of a trait are taken as instances of the same functional trait (be it a token or a type) if they can be integrated into an abstract description of organizational closure. Functional identification results from the very theoretical operation of describing closure.

In Saborido and al. (2011) and Moreno \& Mossio (2015) we placed heavy emphasis on the fact that the same criterion of individuation applies to both intra-generation and crossgeneration function, the difference being uniquely the relevant regime of closure. In particular, we stressed that, for the purpose of ascribing functions, the organizational continuity sustained by closure grounds individuation in spite of other discontinuities that might exist between the various temporal instances. In the case of cross-generation functions, in particular, we wrote:

"The various temporal instances (at time scales $\tau_{1}, \tau_{2} \ldots<\tau_{n}$ ) of a system can be considered - in spite of any changes that may occur - instances of the same encompassing self-maintaining organisation, to the extent that their constitutive organisation realises closure at $\tau_{\mathrm{n}}$. In particular, this implies that the system in which a trait $x$ performs an enabling function at time $\tau_{1}$ is the same system in which, at $\tau_{2}$, that function of $x$ is dependent, if both $\tau_{1}$ and $\tau_{2}$ are included in $\tau_{\mathrm{n}}$ (at which closure is realised). In other terms, for the purposes of ascribing functions, the continuity of closure (and thus the maintenance of the system) takes precedence as a criterion of individuation over other criteria on the basis of which the various instances of the organisation would possibly not be taken as instances of the same system. If there is a causal dependence between two temporal instances of a system, such that their conjunction realises closure, then it could be claimed that, in this respect (and possibly only in this respect) the two instances are temporal instances of the same encompassing organisation". (Moreno \& Mossio, p. 79)

This paragraph can be slightly amended in the light of the preceding discussion about the notion of closure. In particular, the "causal dependence" which is mentioned can be more precisely understood as "constraint dependence": if there is a continuous chain of constraint dependences, and the iteration of a similar pattern that allows folding it up, then instances of the same trait occurring at different generations can be subject to the same closure. According to the OA, hence, cross-generation functional traits are individuated for the same reasons than functional traits in individual organisms. Therefore, if organizational criteria are acceptable and coherent in the latter case (an issue with which we do not deal here), then we submit that they apply also to the former case, in spite of the fact they might seem counterintuitive.

OA adopts a criterion of functional identification based on organizational closure, which implies a continuity of constraint dependences through time. Although its application might be debatable and should of course be the object of accurate philosophical scrutiny, we believe 
that it constitutes a relevant alternative to evolutionary criteria of functional identification at work in selected effects etiological approaches.

\section{References}

Artiga, M. \& Martinez, M. (2016). The Organizational Account of Function is an Etiological Account of Function. Acta Biotheoretica, 64(2), 105-117.

Bigelow, J. \& Pargetter R. (1987). Functions. Journal of Philosophy, 84, 181-196.

Cummins, R. (1975). Functional analysis. Journal of Philosophy, 72, 741-765.

Davies, P. S. (2001). Norms of Nature. Naturalism and the Nature of Functions. MIT Press: Cambridge.

Delancey, C. (2006). Ontology and teleofunctions: A defense and revision of the systematic account of teleological explanation. Synthese, 150, 69-98.

Godfrey-Smith, P. (1994). A modern history theory of functions. Noûs, 2, 344-362.

Millikan, R.G. (1989). In defense of proper functions. Philosophy of Science, 56, 288-302.

Montévil, M., \& Mossio. M. (2015). Biological Organisation As Closure Of Constraints. Journal of Theoretical Biology, 372, 179-191.

Moreno, A. \& Mossio M. (2015). Biological Autonomy. A Philosophical and Theoretical Enquiry. Springer: New York.

Mossio, M. (2013). Closure, causal. In W. Dubitzky, O. Wolkenhauer, K.-H. Cho, \& H. Yokota (Eds.), Encyclopedia of systems biology, Springer: New York, pp. 415-418.

Mossio, M., Saborido, C. and Moreno, A. (2009). An Organizational Account for Biological Functions, British Journal for the Philosophy of Science, 60 (4), 813-841.

Neander, K. (1991). Function as selected effects: The conceptual analyst's defense. Philosophy of Science, 58, 168-184.

Nunes, N., Moreno, A., and El Hani, C. (2014). Function in ecology: an organizational approach. Biology and Philosophy, 29(1), 123-141.

Saborido, C. (2014) New directions in the Philosophy of Biology: a New Taxonomy of Functions. In: C. Galavotti, S. Hartmann, M. Weber, W. Gonzalez, D. Dieks and T. Uebel (ed.) New Directions in the Philosophy of Science. Springer: New York, pp. 235-251.

Saborido, C., Mossio, M., and Moreno, A. (2011). Biological organization and crossgeneration functions. British Journal for the Philosophy of Science, 62 (3), 583-606.

Saborido C. \& Moreno, A. (2015) Biological Pathology from an Organizational Perspective. Theoretical Medicine and Bioethics 36 (1): 83-95.

Walsh, D. M. (1996). Fitness and Function. The British Journal for the Philosophy of Science, 47 (4): 553-574 .

Wright, L. (1973). Functions. Philosophical Review, 82, 139-168. 